\title{
OPEN Detailed characterization of the transcriptome of single B cells in mantle cell lymphoma suggesting a potential use for SOX4
}

Simone Valentin Hansen ${ }^{1 凶}$, Marcus Høy Hansen ${ }^{1}$, Oriane Cédile $^{1,2}$, Michael Boe Møller ${ }^{1}$, Jacob Haaber ${ }^{1}$, Niels Abildgaard ${ }^{1,3}$ \& Charlotte Guldborg Nyvold ${ }^{1,2,3}$

Mantle cell lymphoma (MCL) is a malignancy arising from naive $B$ lymphocytes with common bone marrow (BM) involvement. Although $\mathrm{t}(11 ; 14)$ is a primary event in MCL development, the highly diverse molecular etiology and causal genomic events are still being explored. We investigated the transcriptome of $\mathrm{CD} 19^{+} \mathrm{BM}$ cells from eight MCL patients at single-cell level. The transcriptomes revealed marked heterogeneity across patients, while general homogeneity and clonal continuity was observed within the patients with no clear evidence of subclonal involvement. All patients were SOX $11^{+} C \mathrm{CND} 1^{+} \mathrm{CD} 20^{+}$. Despite monotypic surface immunoglobulin $(\mathrm{Ig}) \mathrm{k}$ or $\lambda$ protein expression in MCL, $10.9 \%$ of the SOX11 + malignant cells expressed both light chain transcripts. The early lymphocyte transcription factor SOX 4 was expressed in a fraction of SOX11 + cells in two patients and co-expressed with the precursor lymphoblastic marker, FAT1, in a blastoid case, suggesting a potential prognostic role. Additionally, SOX 4 was found to identify non-malignant SOX11- pro-/pre-B cell populations. Altogether, the observed expression of markers such as SOX4, CD27, IgA and IgG in the SOX $11^{+} \mathrm{MCL}$ cells, may suggest that the malignant cells are not fixed in the differentiation state of naïve mature $B$ cells, but instead the patients carry $B$ lymphocytes of different differentiation stages.

Mantle cell lymphoma (MCL) is a subtype of non-Hodgkin's lymphoma (NHL) with a generally aggressive although heterogeneous disease course $e^{1,2}$. One of the primary oncogenic events is the $t(11 ; 14)(q 13 ; q 32)$ translocation juxtaposing the cyclin D1 (CCND1) proto-oncogene to the Ig heavy chain (IGH) locus ${ }^{3}$ leading to overexpression of CCND1 and cell cycle deregulation ${ }^{4}$. This translocation is observed in the majority (90\%) of MCL cases ${ }^{1}$, but also CCND1 negative cases have been reported, where patients showed overexpression of CCND2 $2^{5}$ or $\mathrm{CCND}^{6}$. The translocation $\mathrm{t}(11 ; 14)$ is presumably acquired in immature pre-B cells of the bone marrow (BM), although the full oncogenic potential develops in mature $\mathrm{B}$ cells ${ }^{2}$. The typical immunophenotype is surface expression of CD19, CD20, CD22, CD43, CD79a, CD5 and FMC7 with monoclonal $\mathrm{k} / \lambda$ immunoglobulin (Ig) light chains, while CD23 (also known as FCER2), CD10 (also known as MME), CD200 and BCL6 are typically dim or negative $e^{1,2,7}$. In the development of B cells the IGH locus undergoes V(D)J rearrangement forming a unique $B$ cell receptor ${ }^{8}$. As MCL raises from one cell of origin with a unique $V(D) J$ rearrangement, this rearrangement is characteristic for the malignant clone and can be used as a fingerprint for tracking malignant cells ${ }^{9}$.

The development of MCL directs into two major biological and clinical variants; classical nodal MCL and leukemic non-nodal MCL ${ }^{2,10,11}$. Classical MCL has usually an aggressive clinical course and typically involves lymph nodes and other extra-nodal sites at presentation. This form presents with a higher degree of genomic instability $2,10,12$, and is positive for SOX11, an acknowledged specific marker of MCL ${ }^{13,14}$. This subtype originates in a B cell that is unexposed to the germinal center and therefore has no or low percentage of IGHV somatic hypermutations and an epigenetic methylation signature, corresponding to naive $\mathrm{B}$ cells ${ }^{2}$. The acquisition of

${ }^{1}$ Hematology-Pathology Research Laboratory, Research Unit for Hematology and Research Unit for Pathology, University of Southern Denmark and Odense University Hospital, Odense, Denmark. ${ }^{2}$ OPEN, Odense Patient Data Explorative Network, Odense University Hospital, Odense, Denmark. ${ }^{3} \mathrm{CITCO}$, Centre for Cellular Immune Therapy of Hematological Cancer Odense, Odense University Hospital, Odense, Denmark. ${ }^{\circledR}$ email: simone.valentin.hansen@rsyd.dk 
additional molecular aberrations can lead to more aggressive variants ${ }^{2,10,12}$. Leukemic non-nodal MCL is negative for SOX11 and typically involves peripheral blood (PB), BM, and spleen ${ }^{2,10,15,16}$. This subtype originates in a B cell that has been exposed to the germinal center and therefore has hypermutated IGHV and a methylation signature corresponding to memory B cells ${ }^{2}$. These cases are often clinically indolent with superior outcome compared to classical MCL, but may evolve to aggressive disease when additional aberrations occur ${ }^{2,10,15,16}$. Classical MCL is the most common type, while leukemic non-nodal MCL represents only $10-20 \%$ of patients ${ }^{16}$. Histological variants include classic MCL with monomorphic lymphoid proliferation of small to medium sized cells, where the proliferative activity usually is low ${ }^{2}$. More aggressive types include the blastoid and the pleomorphic variants ${ }^{2}$, which constitute $10 \%{ }^{17}$ to $>20 \%$ of all MCLs ${ }^{18}$, respectively.

SOX11 is a member of the SOXC protein family, which also includes SOX4 and SOX $12^{19}$. The three SOXC proteins exhibit overlapping expression patterns and molecular properties, and may act in redundancy to control developmental, physiological and pathological processes ${ }^{19-22}$. SOX11 is a transcription factor that has been reported to promote angiogenesis ${ }^{23}$, migration and adhesion of MCL cells to stromal cells ${ }^{24}$, thereby promoting cell-adhesion-mediated drug resistance ${ }^{24}$. It can impact MCL cells by augmentation of BCR signaling ${ }^{25}$, suppression of $\mathrm{BCL}^{26}$ to avoid MCL cells entering the germinal center thereby keeping IGHV unmutated, and by activation of PAX-5 thereby blocking the maturation to plasma cell ${ }^{27}$. SOX 4 is a homologous transcription factor $^{19,22}$ required for development and differentiation of lymphocytes ${ }^{28-30}$ and was found to be expressed in pro-B cells ${ }^{31}$. In acute myeloid leukemia, SOX4 was shown to be an important factor in leukemogenesis ${ }^{32,33}$, and high expression of SOX 4 was a poor prognostic factor ${ }^{32}$. In pre-B acute lymphoblastic leukemia, SOX4 was found to be required for survival, progression and proliferation ${ }^{34,35}$ and correlates with poor clinical outcome out,35. $^{34}$. Elevated SOX 4 expression has also been found in a wide variety of solid cancers, where mostly oncogenic roles have been reported ${ }^{22,36}$.

Collectively, MCL is considered a highly heterogeneous disease with respect to clinical presentation and prognosis $^{37,38}$, and high molecular variation with subclonal intra-tumor heterogeneity has been demonstrated already at diagnosis ${ }^{39-41}$. Presence of multiple subclones at diagnosis has been associated with decreased relapsefree survival, suggesting a prognostic impact ${ }^{42,43}$. The molecular heterogeneity of MCL makes it challenging to define standard therapies ${ }^{12}$ and is a plausible explanation for the diverse outcomes of this B malignancy. In this study, we investigated the transcriptome of MCL cells from eight diagnostic BM samples to provide insight into the complex and diverse molecular architecture of MCL at the single-cell transcriptomic level in the perspective of commonly used molecular pathology markers.

\section{Results}

Single cell RNA-sequencing (scRNA-seq) was performed on $\mathrm{CD}_{19} 9^{+} \mathrm{B}$ lymphocytes isolated from diagnostic bone marrow aspirates (Fig. S1 and Table S1) of eight patients diagnosed with MCL. A total of 30,565 cells were collected using the Chromium platform. On average, 3800 cells from each patient passed the quality threshold and were included in the downstream analyses (1018-6668 cells, Table S2). In general, patient samples 2, 4, and 6 displayed superior quality relative to the rest of the cohort, with a median of 772-1151 expressed genes per cell versus 309-517 (Table S2). Of note, the quality of the sequencing output was in concordance with higher clonal infiltration of bone marrow, cell purity and RNA integrity (Table S1-S3).

Global transcriptomic profiles of MCL bone marrow B lymphocytes. Joint dimensional reduction, using UMAP (Uniform Manifold Approximation and Projection ${ }^{44}$ ) of the single cell transcriptomes to low dimensional feature space showed a resolution to discern discrete transcriptomic populations of the individual cases (Fig. 1A). This transcriptomic heterogeneity was in concordance with the general notion of inter-patient heterogeneity of MCL. A significant correlation was found between SOX11 expression $\left(p=0.003, \mathrm{R}^{2}=0.996\right.$, Fig. 1B) and molecular pathology markers frequently applied in diagnosis of MCL, whereas SOX4 negatively correlated with these markers.

Expression of molecular pathology markers frequently applied in diagnosis of $\mathrm{MCL}$ at single cell transcriptomic level. Concordant with the clinical laboratory results, all patients were positive for SOX11 and CCND1, while only 37.5\% of the total single-cell population expressed SOX11 at a detectable level (ranging from 9.8 to $64.6 \%$ in the individual patients, Figs. 1C, 2, Table S4-S5), and 71.1\% (range: 26.4-81.4\%) of the SOX $11^{+}$cells co-expressed CCND1 (Table S5). Looking into the two other homologous SOXC family members, $4.4 \%$ of all cells expressed SOX4 (range: $0.4-16.0 \%$ ), while being negative for SOX12 (Figs. 1C, 2, Table S4S5). Three patients $(1,3$ and 7$)$ harbored a substantial SOX4 positive fraction within the SOX11 expressing cells of $19.7 \%, 13.3 \%$ and $12.2 \%$, respectively (Figs. 1C, 2, Table S4-S5).

Generally, the combined population was positive for CD20 and did not express the transcripts for CD5, CD19, CD23 or CD27 (Figs. 1C, 2, Table S4-S5), although the profiles varied patient-wise. Patient 5 was almost completely devoid of measurable CD5 and CD19 transcripts in the SOX11 ${ }^{+}$population (Table S5). $10.8 \%$ of all cells (range: $0.2-24.5 \%$ ), and $10.9 \%$ of SOX $11^{+}$cells (range: $0.3-25.8 \%$ ), were found positive for both $\kappa$ and $\lambda \mathrm{Ig}$ light chain genes (Figs. 1C, 2, Table S4-S5). While $90.5 \%$ of all SOX $11^{+}$cells were positive for IgM (range: $51.6-98.7 \%$, Table S5), only $17.8 \%$ expressed IgD (range: $0.8-43.1 \%$, Table S5). All patients harbored SOX1 $1^{+}$cells expressing IgA (range: $6.1-35.1 \%$, Table S5) and IgG (range: $0.8-14.6 \%$, Table S5), and a small SOX $11^{+} \mathrm{CD} 27^{+}$fraction (3-9.6\%, Table S5) was detected in patient 2,4 and 6 . Additionally, minor compartments of SOX $11^{+} \mathrm{CD} 23^{+}$cells were detected in all patients (range: $1.4-9.2 \%$, Table S5).

Differential expression analysis with gene set enrichment analysis (GSEA, data not shown) identified non-malignant pro-/pre-B cells within the cohort significantly different from the malignant and SOX $11^{+}$ cells. These cells were enriched in bone marrow pre-B markers (GSEA, marrow CD $34^{+}$pre- $\mathrm{B}^{45}, p=6.9^{*} 10^{-63}$, 
A

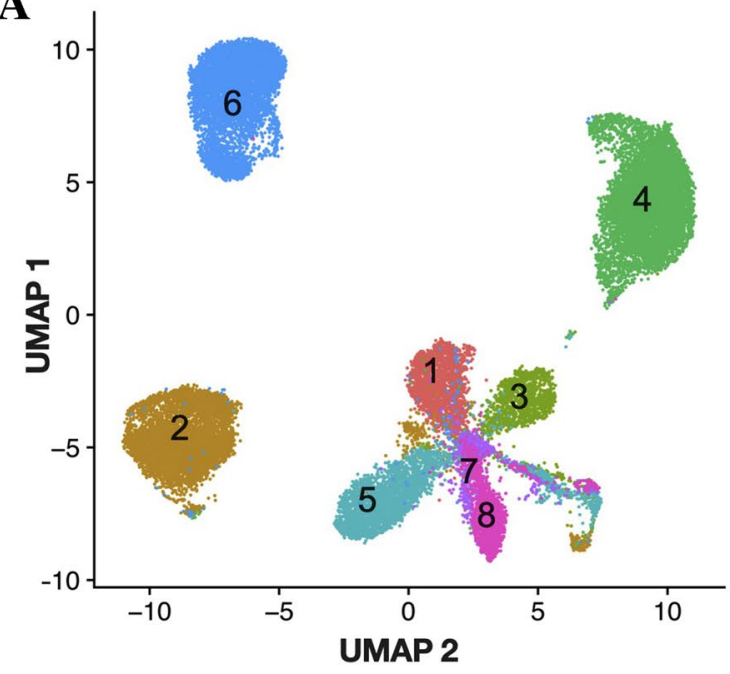

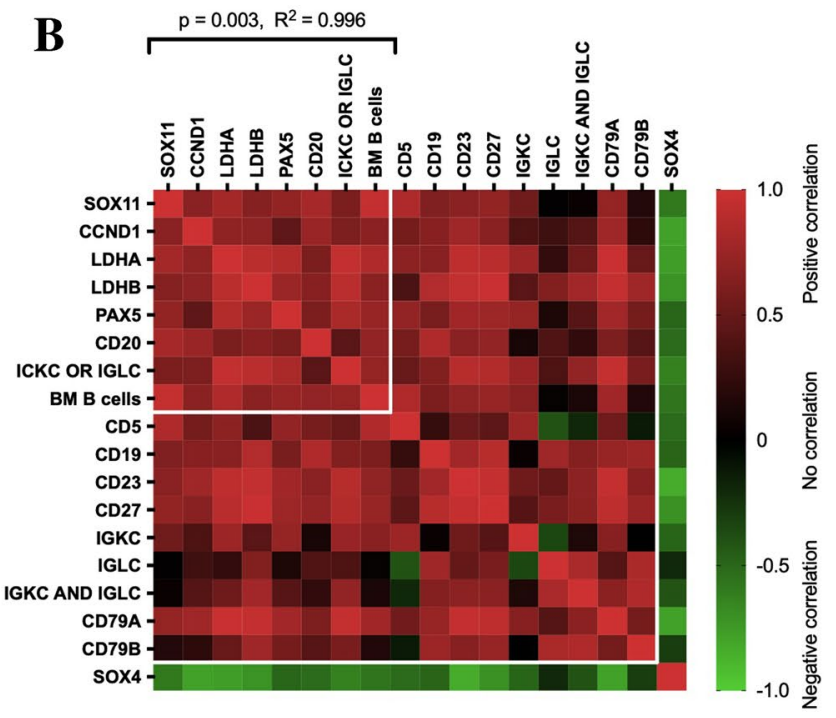

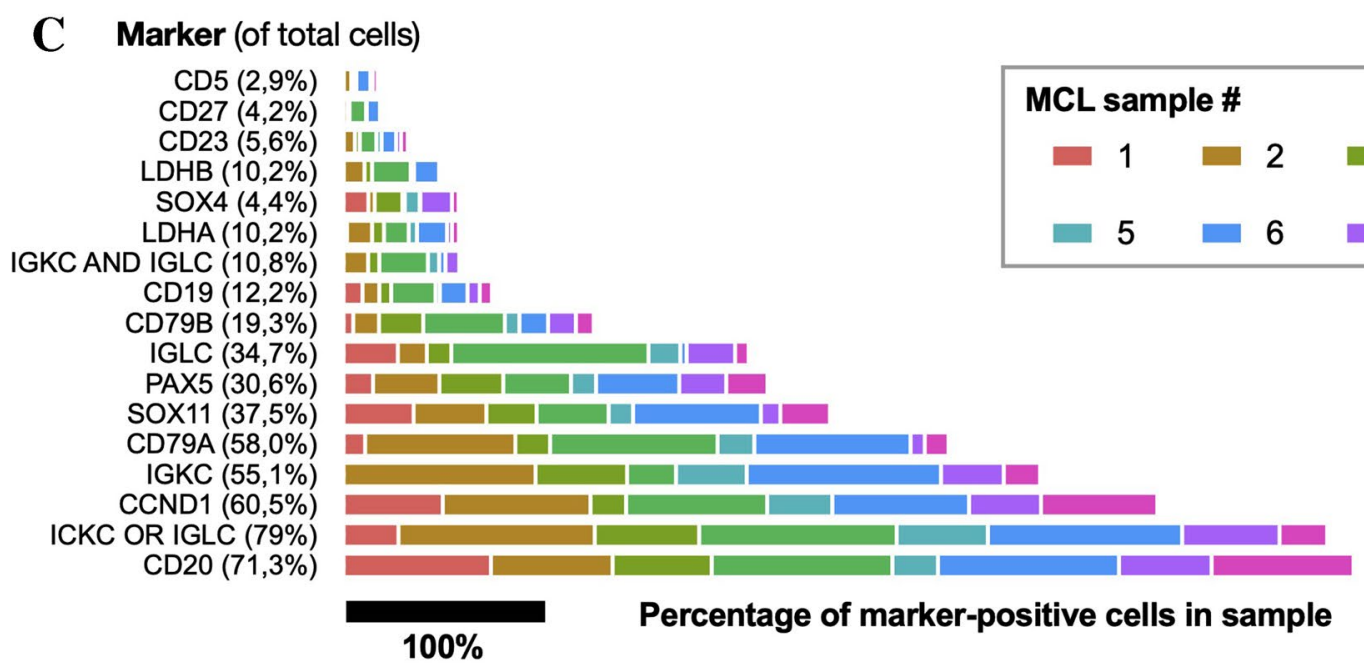

Figure 1. Clustering of combined single-cell transcriptomes, expression and correlation of molecular pathology MCL markers. The MCL cohort displayed heterogeneous expression profiles, forming distinct clusters of single-cell transcriptomes, except for co-located sample 7 and 8. Cells are colored and numbered according to patient origin (A). A highly significant positive correlation (red) was found between the percentage of SOX11 expressing cells and cells expressing frequently used molecular pathology markers in MCL (B) including CCND1, LDHA/B, PAX5, CD20, immunoglobulin $\kappa / \lambda$ light chain (IGKC/IGLC) $(p=0.003)$. The fraction of SOX11 positive cells also showed a positive correlation with the percentage of $B$ cells in the bone marrow samples known from the clinical flow cytometry analysis ( $B M B$ cells, $p=0.003)$. The rest of the listed markers showed strong multicollinearity and were hence excluded in the linear regression. The percentage of SOX $4^{+}$cells was negatively correlated (green), with other markers and associated with residual non-malignant pro-/pre-B cells (see also Fig. 2b). The percentage of cells expressing molecular pathology markers were calculated for the individual patients (bars) and from the total single cell cohort (numbers) as described in the methods section (C). The total single cell population (numbers) and the cells from individual patients (bars) were markedly positive for CCND1, CD20 and $\kappa / \lambda$ (IGKC/IGLC), while merely $37.5 \%$ of the total sequenced cells were SOX $11^{+}$, varying from 9.8 to $64.6 \% .10 .8 \%$ of the combined cohort was found to harbor dual expression of $\kappa$ and $\lambda$ light chains.

$\mathrm{q}_{\mathrm{FDR}}=4.81^{\star} 10^{-59}, 40 / 98$ gene overlap) and markers of lymphocyte progenitors $\left(\mathrm{GSEA}^{46}, p=3.33^{\star} 10^{-43}\right.$, $\mathrm{q}_{\mathrm{FDR}}=1.16^{\star} 10^{-39}, 41 / 289$ gene overlap) such as SOX4, IGLL1 (also known as IGL5/CD179B/VPREB2), DNTT, VPREB1, and CD10 (Fig. 2B). As expected, the non-malignant B cells were co-localized within the cohort by transcriptional clustering and did not show evidence of Ig light chain restriction (Figs. 1A, 2).

Local transcriptomic profiles of malignant cells. Next, we explored how expression profiles varied among purified $\mathrm{CD} 19^{+}$bone marrow cells within the individual patients. The most frequent significantly altered 
A

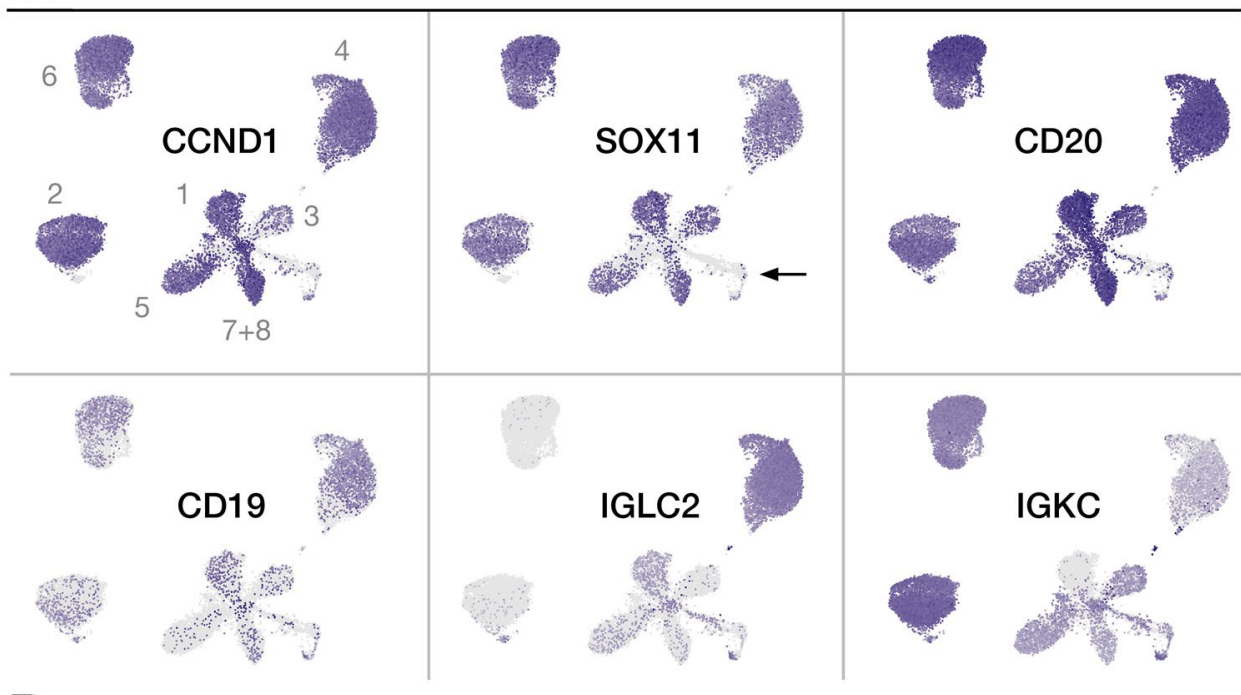

$\underline{B}$

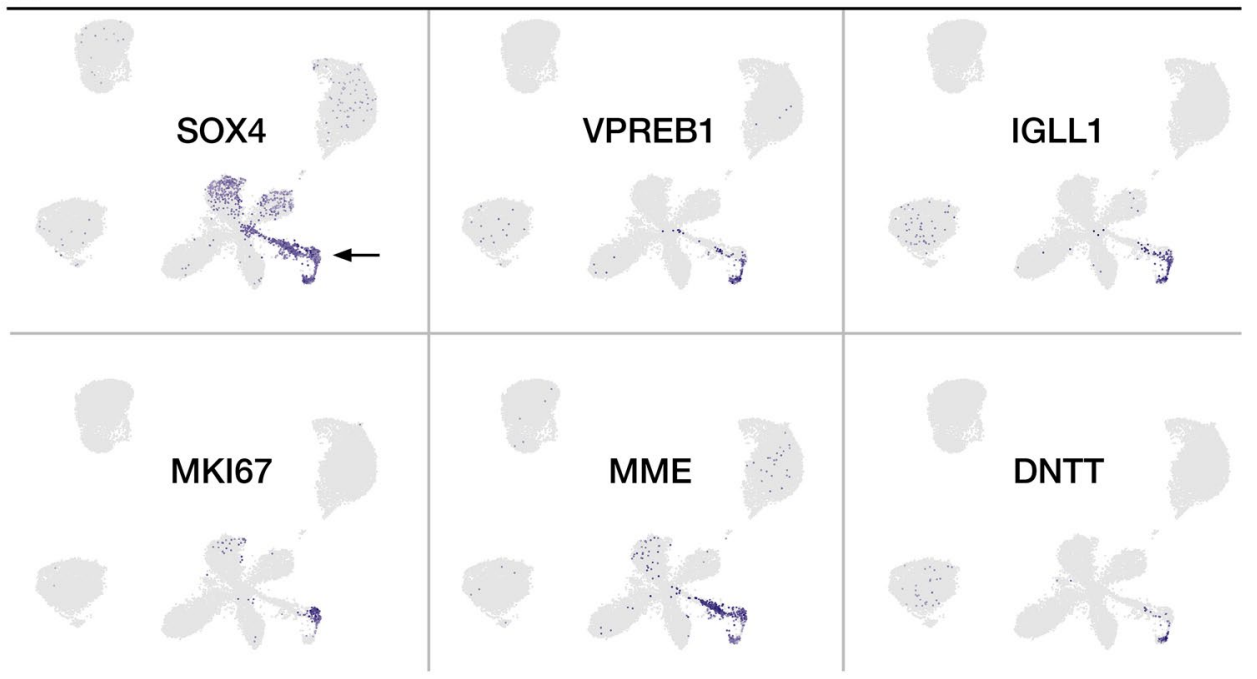

Figure 2. Global expression of molecular pathology markers frequently applied in diagnosis of mantle cell lymphoma (A) and markers associated with immature B cells (B) in combined mantle cell lymphoma (MCL) single cell transcriptomes. The resolved representations of cells from each MCL patient (pt.) were in general agreement with molecular pathology markers of MCL (A), although CD19 was only positive in a fraction of the single cells at the transcriptional level (12.2\%). The dominating Ig $\kappa$ and $\lambda$ light chain restriction could be transcriptionally identified, with pt. $2,3,5,6,7,8$ being $\kappa$, and 1 and 4 being $\lambda$, the average double-positive was $7 \%$, ranging from 0 (pt. 1) to $24 \%$ (pt. 4). CCND1 and CD20 were the most widely expressed molecular pathology markers. Markers of immature pre-pro B cells and pro-B cells were expressed in SOX11 ${ }^{-}$areas containing cells from multiple patients (B). Three patients (pt. 1, 3 and 7) had a substantial cell fraction expressing SOX4 co-localized with SOX $11^{+}$. Purple indicates positive expression, and the intensity of the color reflects increased expression. MME: also known as CD10.

genes from unsupervised clustering (Seurat cluster resolution 0.2-0.4, data not shown) were related to NFkB signaling (14 genes), apoptosis (9 genes), IL2/STAT5 (5 genes) and TP53 pathways (5 genes) (GSEA, hallmark gene sets, $\left.5.39^{\star} 10^{-5}>p>4.7^{\star} 10^{-18}, 3.85^{\star} 10^{-4}>\mathrm{q}_{\mathrm{FDR}}>2.35^{\star} 10^{-16}\right)$.

Algorithmically defined clusters (shared nearest neighbor (SNN) clustering) of each patient did not provide any clear evidence of multiple clones or subclones within the malignant population, with the exception of Patient 2 (Fig. 3). The general lack of multiple clones and subclones was supported by subsequent deep sequencing of immunoglobulin heavy chain gene rearrangements (data not shown) using the LymphoTrack assay [704,889 mapped IgH reads $(537,282-858,000)]$. Except for patient 2 , all eight patients were found to have a single malignant $\mathrm{B}$ cell clone since only one $\mathrm{V}(\mathrm{D}) \mathrm{J}$ rearrangement was detected by deep sequencing of immunoglobulin heavy chain gene rearrangements (data not shown). In patient 2, two different rearrangements with different J genes was found. Although the minor clone only constituted $\sim 1 \%$, this was indicative of two different B cell clones in 
A
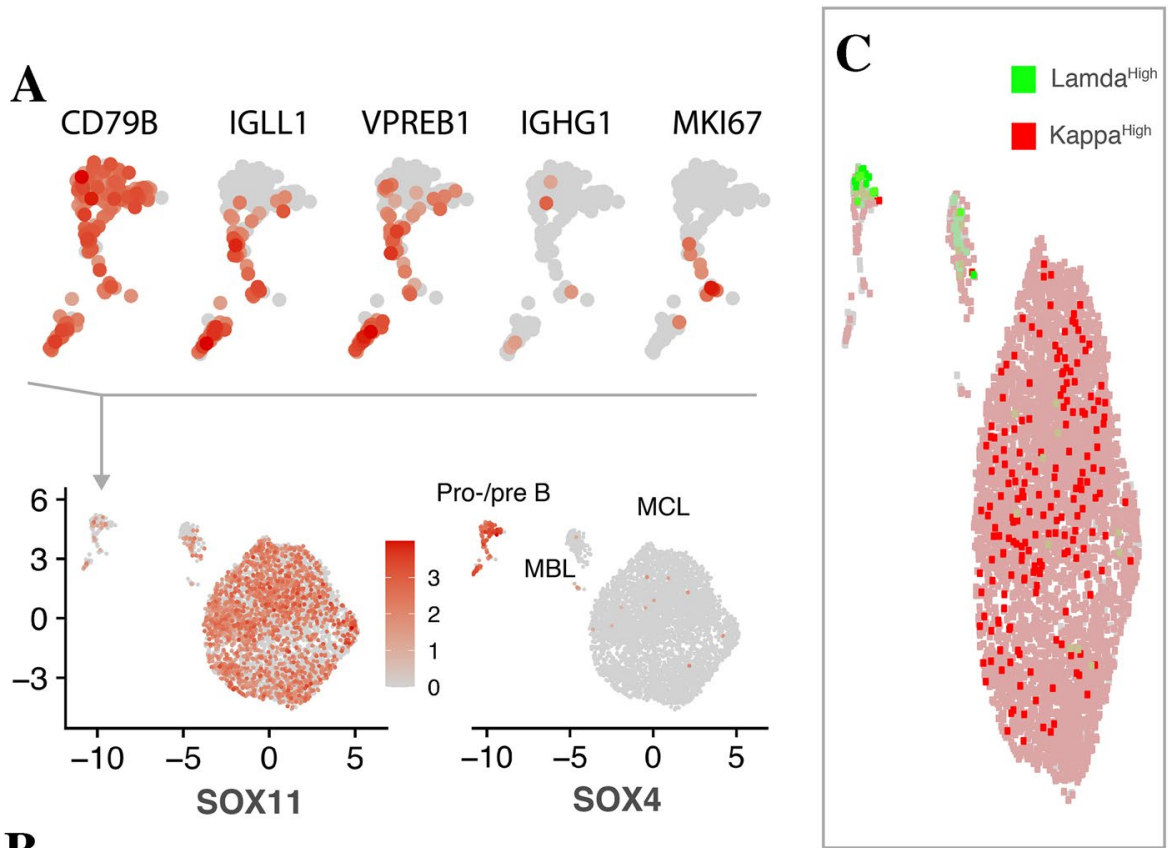

B

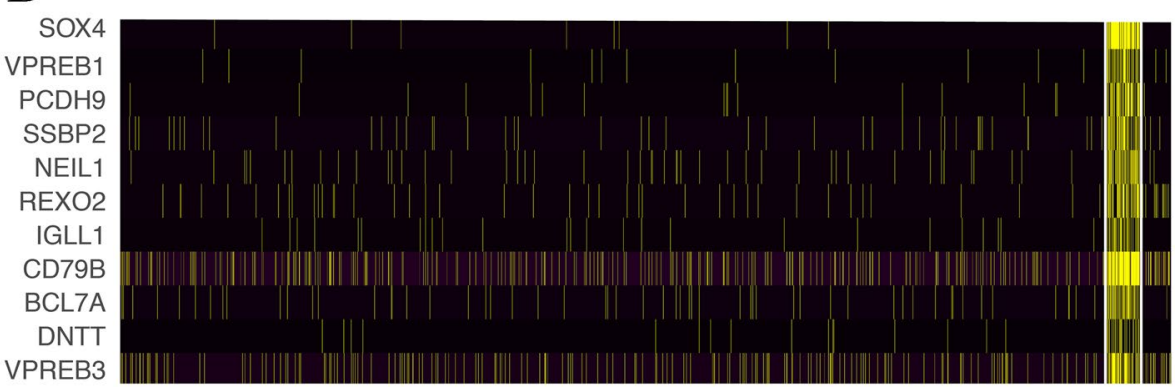

Figure 3. Cluster trio of patient 2. The resolved subpopulations could be identified as a large cohort of lymphoma cells (MCL), a minor subpopulation assumed to reflect monoclonal B cell lymphocytosis (MBL), and a relatively small cluster of cells expressing (red) IGLL1 with a possible role in pro-B cell to pre-B cell differentiation, with little evidence of isotype-switched B cells (Pro/pre B) (A). The profile of this cluster was significant for immature B cells of either pro- or pre-B cells (yellow indicates positive expression) (B). Importantly, immature SOX $11^{+}$cells was discernible. The minor population assumed to represent an MBL clone, resolved from flow cytometry, was positive for both immunoglobulin light chain $\lambda$ (green) and $\kappa$ (red) genes (IGLC, IGKC) as was a fraction of immature cells (C), while the MCL clone was $\lambda$ negative. Also, the MBL cluster was enriched in CD23 and markers of isotype-switched B cells (IgA and IgG) (not shown).

this patient, and may be in consistence with this patient having a small monoclonal B cell lymphocytosis (MBL) clone according to the clinical flow data (Table 1). Subclone analysis based on somatic hypermutation showed no clear evidence of subclonal evolution in any of the samples.

The two distinct subclusters of Patient 2 (Fig. 3A) were identified as one expressing markers of immature B cells (pro/pre-B cells, Fig. $3 \mathrm{~B}$ ), and the other suggestive of MBL with a $\lambda$ positive CLL-like profile in line with the clinical flow cytometry data from this patient (Table 1 ). This cluster, constituting $\sim 3.3 \%$ of the cells, was significantly increased for Ig light chain $\lambda$ genes (Fig. 3C, IGLC1, IGLC2, 3.6-12 $\times$ fold-change, $58.1-75 \%$ positive cells in this cluster versus $2.4-8.8 \%$ in other clusters), CD23 $(2.7 \times$ fold-change, $42 \%$ positive cells in this cluster vs $5 \%$ in other clusters) and isotype-switched B markers (IgG, IgA) along with MEF2C, FCRL1 and other B cell markers. Although the generated clusters were strongly indicative of pro/pre-B cells and MBL, respectively, both contained a small and partly SOX11 positive cell subset (13\%, $2.5 \times$ expressional decrease).

Apart from the results related to Patient 2, one of the most significant findings from the entire cohort of malignant SOX $11^{+}$cells was the identification of distinct markers from blastoid MCL cells of Patient 1, e.g. protocadherin FAT1 expressing cells (Fig. 4). FAT1, almost exclusively located in the bone marrow B lymphocytes of patient 1, was expressed in a compartment of SOX4, Aryl Hydrocarbon Receptor (AHR), Chromodomain Helicase DNA Binding Protein 3 (CHD3) and Dystonin (DST) positive cells (Fig. 4B). The blastoid case was evidently monoclonal, $\lambda$ chain restricted, with a very small but identifiable number of malignant MKI67 expressing cells (data not shown). We did not observe any informative individual features in the rest of the cohort. 


\begin{tabular}{|c|c|c|c|c|c|c|c|c|}
\hline Patient & 1 & $2^{\mathrm{a}}$ & 3 & 4 & 5 & 6 & 7 & 8 \\
\hline Sex & Female & Female & Male & Male & Male & Female & Male & Male \\
\hline Age & 86 & 72 & 78 & 62 & 67 & 72 & 68 & 88 \\
\hline Date of diagnosis & July 2017 & April 2018 & May 2017 & Nov 2017 & Nov 2017 & July 2017 & May 2019 & Oct 2019 \\
\hline $\begin{array}{l}\text { Infiltration of MCL } \\
\text { in bone marrow (\% } \\
\text { of vital cells) }\end{array}$ & $28 \%$ & $38.2 \%$ & $\begin{array}{l}11.6 \% \text { (based on } \\
\text { previous sample) }\end{array}$ & $\begin{array}{l}32 \% \text { (based on } \\
\text { previous sample) }\end{array}$ & $4.3 \%$ & $71.2 \%$ & $4.6 \%$ & $6.08 \%$ \\
\hline $\begin{array}{l}\text { Fraction of MCL } \\
\text { cells from CD19 } \\
\text { cells in bone marrow }\end{array}$ & $100 \%$ & $93.6 \%$ & $88.3 \%$ & $95.5 \%$ & $100 \%$ & $100 \%$ & $82.8 \%$ & $57.5 \%$ \\
\hline Nodal involvement & Yes & Yes & Yes & Yes & Yes & Yes & Yes & Yes \\
\hline Splenomegaly & No & No & Yes & Yes & No & No & No & No \\
\hline Extra-nodal sites & Lungs & Coecum & No & No & No & Pleural fluid & Tongue & No \\
\hline $\begin{array}{l}\text { Leukocyte count in } \\
\text { blood }\end{array}$ & $17.4^{*} 10^{9} / \mathrm{L}$ & $16^{*} 10^{9} / \mathrm{L}$ & $12 * 10^{9} / \mathrm{L}$ & - & - & $18.4^{*} 10^{9} / \mathrm{L}$ & $6.17^{*} 10^{9} / \mathrm{L}$ & $8.48^{*} 10^{9} / \mathrm{L}$ \\
\hline Morphology & Blastoid & Classical & - & Classical & Pleomorphic & Classical & Classical & Pleomorphic \\
\hline Cyclin D1 ${ }^{+}$ & Yes & Yes & Yes & Yes & Yes & Yes & Yes & Yes \\
\hline SOX11 $^{+}$ & Yes & Yes & $40 \%$ & Yes & Yes & Yes & Variable & Yes \\
\hline Immuno-phenotype & $\begin{array}{l}\mathrm{CD} 19^{+} \mathrm{CD} 20^{+} \\
\mathrm{CD} 22^{+} \lambda^{\text {thigh }} \mathrm{CD} 5^{+} \\
\mathrm{CD} 79 \mathrm{~b}^{+} \\
\mathrm{CD} 10^{\text {partly }}\end{array}$ & \begin{tabular}{|l}
$\mathrm{CD} 19^{+} \mathrm{CD} 20^{+}$ \\
$\mathrm{CD} 22^{\operatorname{dim}}$ \\
$\mathrm{\kappa}^{+}$ \\
$\mathrm{CD}^{+}$
\end{tabular} & $\begin{array}{l}\mathrm{CD} 19^{+} \mathrm{CD} 20^{+} \\
\mathrm{k}^{\text {kigh }} \\
\mathrm{CD}^{\mathrm{C}} 7 \mathrm{~b}^{+} \\
\mathrm{CD} 23^{\text {partly }} \\
\mathrm{CD} 22^{+}\end{array}$ & $\begin{array}{l}\mathrm{CD} 19^{+} \mathrm{CD} 20^{+} \\
\mathrm{CD} 22^{+} \\
\lambda^{+} \mathrm{CD} 5^{\mathrm{dim}} \mathrm{CD}^{2} 9 \mathrm{~b}^{+} \\
\mathrm{CD} 27^{\mathrm{dim}}\end{array}$ & $\begin{array}{l}\mathrm{CD} 19^{+} \\
\mathrm{CD} 20^{+} \\
\mathrm{CD} 22^{+} \\
\kappa^{+}\end{array}$ & $\begin{array}{l}\mathrm{CD}^{2} 5^{+} \\
\mathrm{CD} 19^{+} \mathrm{CD} 20^{+} \\
\text {CD22 } \\
\text { CD7im }^{\text {high }}{ }^{+}\end{array}$ & $\begin{array}{l}\mathrm{CD} 19^{+} \mathrm{CD} 20^{+} \\
\mathrm{CD}^{+} \\
\mathrm{\kappa}^{+}\end{array}$ & $\begin{array}{l}\mathrm{CD} 19^{+} \\
\mathrm{CD} 20^{+} \\
\mathrm{CD}^{+} \\
\kappa^{+} \\
\mathrm{CD} 38^{+}\end{array}$ \\
\hline $\begin{array}{l}\text { MIPIc score at } \\
\text { diagnosis }\end{array}$ & 8.5 & 7.1 & 7.2 & 6.3 & 8.4 & 7.3 & 5.8 & $7.8 \#$ \\
\hline Current status & Dead from MCL & Alive & $\begin{array}{l}\text { Dead (not from } \\
\text { MCL) }\end{array}$ & Alive & Alive & Alive & Alive & Alive \\
\hline Time to progression & $\begin{array}{l}1 \text { st relapse: } \\
10 \text { months } \\
\text { No response to 2nd } \\
\text { line therapy }\end{array}$ & Not reached & Not reached & Not reached & Not reached & Not reached & Not reached & Not reached \\
\hline Follow-up time & 15 months & 36 months & 47 months & 41 months & 41 months & 45 months & 23 months & 18 months \\
\hline
\end{tabular}

Table 1. Clinical patient information. Patient information was obtained from the clinical records. MCL infiltration was determined by flow cytometry analysis of bone marrow (BM) cells and calculated as percentage of the vital cells in the sample. The fraction of MCL cells from $\mathrm{CD} 19^{+}$cells was calculated, based on the flow cytometry clinical laboratory data, by dividing the percentage of all CD19+ cells including MCL cells, non-malignant B cells and plasma cells ( $\sim 70 \%$ of plasma cells weakly express CD19) by the percentage of monoclonal CD19+ cells in the BM sample. In all patients, the MCL cells were positive for CD19 and CD20 and showed immunoglobulin light chain restriction. All patients had nodal involvement, bone marrow involvement, and were positive for both cyclin D1 and SOX11 as evaluated by immunohistochemistry staining of lymphocytes in lymph node and bone marrow biopsies. Apart from this, the patients were, in consistence with the pathology of MCL, heterogeneous in their clinical presentation. ${ }^{\text {a Patient }} 2$ had a small (2\%) monoclonal B cell lymphocytosis (MBL) clone with a chronic lymphocytic leukaemia (CLL)-like profile $\mathrm{CD} 19^{+} \mathrm{CD} 22^{+} \mathrm{CD} 20^{\mathrm{dim}} \mathrm{CD}^{+}{ }^{+}$ambda ${ }^{\mathrm{dim}}$. \#LDH was unsure.

\section{Discussion}

The complex and diverse molecular architecture of MCL is a plausible explanation for the diverse outcome of the disease. However, it is still unclear what cellular architecture is comprised within the patients. To gain insight into this heterogeneity at single cell level, we performed single cell mRNA sequencing of the purified CD19 ${ }^{+}$ fraction of diagnostic bone marrow aspirates from eight MCL patients. The inter-tumor heterogeneity was striking as previously reported in $\mathrm{MCL}^{47}$. However, in contrast to the subclonal involvement, as shown on unsorted mononuclear cells by two recent scRNA-seq studies ${ }^{47,48}$, the transcriptional profiles observed in this study were rather unremarkable with a homogeneous continuum of expression patterns observed for the malignant cells.

CD19 and CD5 expressions were not detected in all cells at the transcriptional level, as reported previously ${ }^{48}$, indicating a relatively low mRNA abundance or a poor correlation of the proteins and mRNA. Neither CCND1 nor SOX11 was expressed in all malignant B cells from MCL patients and not all SOX $11^{+}$cells expressed CCND1, as observed previously ${ }^{47}$. Collectively, this phenomenon may be explained by transcriptional bursting ${ }^{49-51}$ or simply that the expression levels of the genes were below the detection limit or resolution of the scRNA-seq assay.

We noted that the commonly used markers, expression of $\kappa$ and $\lambda$ Ig light chains, were found to be suboptimal for clonal identification at single cell mRNA level, since co-expression of the transcripts was detected in $10.9 \%$ of the SOX $11^{+}$single cell population, although largely concordant with the light chain restriction observed in the clinical laboratory analyses. The limitations in the number of recorded cells, the panel design (optimal for diagnosis but not for $\kappa$ and $\lambda$ co-expression), and difference in cell preparation used for the diagnosis staining did not enable us to confirm the $\kappa / \lambda$ protein co-expression in the patient clinical flow cytometry data. Previous studies have reported that dual protein expression of $\kappa$ and $\lambda$ Ig light chains could be demonstrated in B cell malignancies ${ }^{52,53}$ and in healthy $B$ cells ${ }^{54,55}$. These observations suggest that this phenomenon is not rare ${ }^{53}$, at 
A
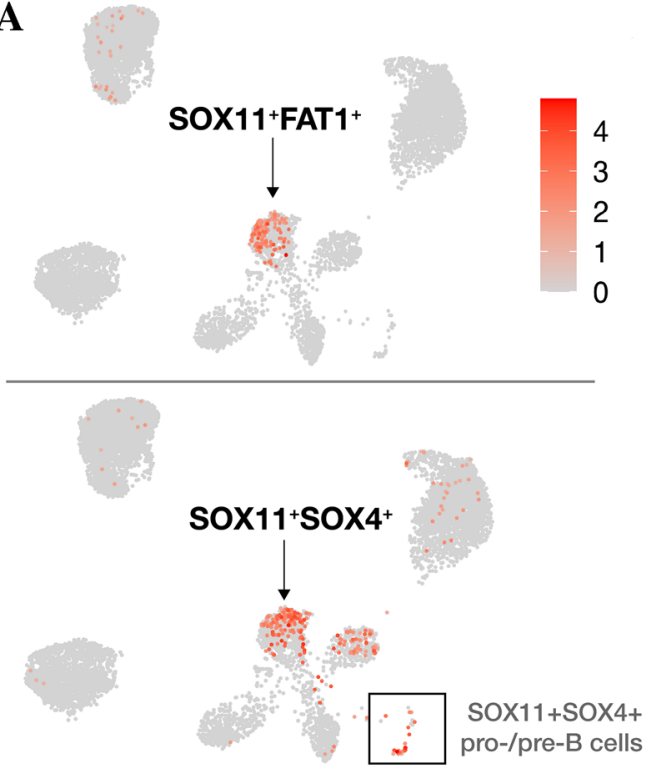

B Blastoid
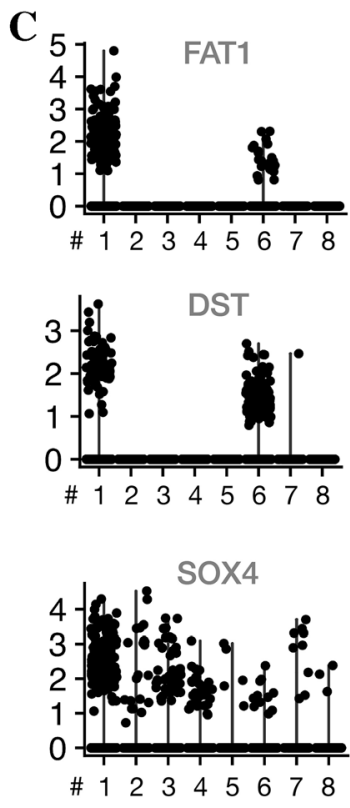

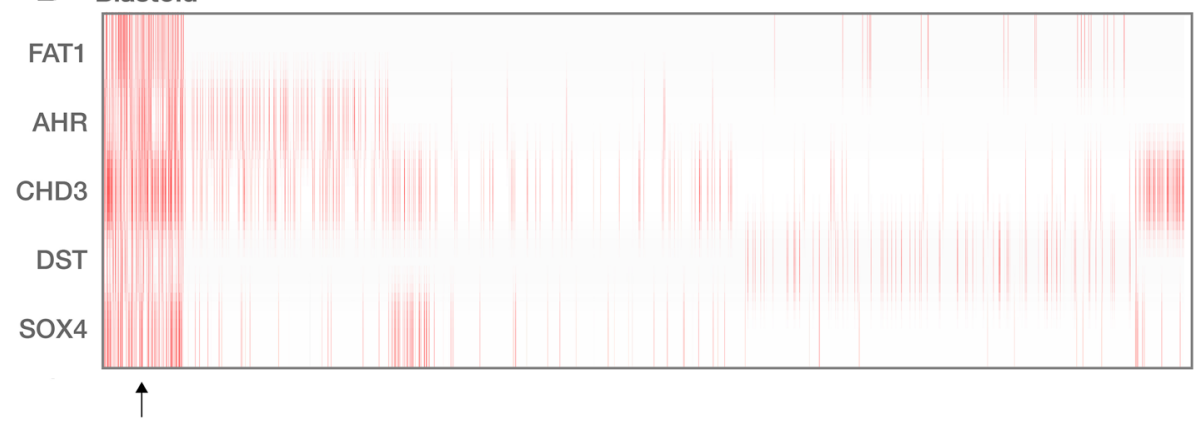

Figure 4. Expression signature of the blastoid mantle cell lymphoma case. Patient 1 had highly specific FAT1 expression (red, upper) among SOX $11^{+}$cells, while SOX4 expression (red, lower) was also found in pt. 3, 4, and 7 as well as in a small fraction of pro-/pre-B cells (A). A large fraction of these cells was also significant for an $\mathrm{AHR}^{+}, \mathrm{CHD}^{+}, \mathrm{DST}^{+}, \mathrm{SOX}_{4}^{+}$expression (red) signature (B), providing potential evidence of a more immature cell type based on previous findings in lymphoblastic leukemia/lymphoma. While the blastoid patient had a very high fraction of malignant SOX $4^{+}$cells, comprising $19.9 \%$ of the SOX $11^{+}$population and no evidence of healthy pro-/pre-B cells, it was not specific for SOX $4^{+}$cells in general. Violin plots show the expression (y-axis) of genes in each patient (x-axis) $(\mathbf{C})$.

least in MCL, and should be considered accordingly, when assessing the clonal burden by means of $\kappa / \lambda$ transcript ratios. It may also suggest that some MCL cells further rearrange Ig light chain genes, or that some of the MCL cells may originate from immature B cells with dual expression ${ }^{52}$.

Not surprisingly, the expression levels of the classical molecular pathology markers used in MCL diagnostics, e.g. SOX11, CCND1, PAX5, CD79B and CD20 were correlated. Although the percentage of measurable CD19 and CD5 positive cells was low, the fractions showed positive correlation with the other markers, whereas a negative correlation was observed between SOX4 and SOX11 in the combined BM B lymphocyte population. This was ascribed to the presence of healthy pro-/pre-B cells, in spite of three patients (patient 1, 3 and 7) coexpressing the transcripts of both of the SOXC proteins. Unexpectedly, the memory B cell marker CD27 and the CLL marker CD23 positively correlated with diagnostic MCL markers. We observed that CD23, frequently used to differentiate CLL from MCL, was present in a subset of the SOX $11^{+} \mathrm{MCL}$ cells, supporting previous findings that some MCLs are CD23 $3^{+56,57}$. The majority of cells were CD20 ${ }^{+} \mathrm{CD} 27^{-}$indicating that few or no memory $\mathrm{B}$ cells were present. In the same line, most cells expressed IgM, indicating mainly naïve mature B cells, concordant with that of $\mathrm{CD} 19^{+}$bone marrow cells and MCL cells of the nodal type.

All patients had SOX $11^{+}$cells expressing transcripts of isotype-switched $\operatorname{IgH}$ in addition to a small $\mathrm{CD} 27^{+} \mathrm{SOX} 11^{+}$fraction observed in patient 2,4 and 6 suggesting that some MCL cells may potentially be antigenexperienced, although expected to originate from naïve B cells. In line with this observation, MCL cells expressing CD27 protein, and transcripts for IgA and have been previously reported ${ }^{58-61}$, in addition to sporadic accounts of $\operatorname{IgA}^{60}$ and IgG surface protein expression ${ }^{61}$. In CLL, resembling MCL in several ways, cells expressing IgG and IgA transcripts with a $\mathrm{V}(\mathrm{D}) \mathrm{J}$ rearrangement identical to that of the IgM+ clone were observed but these cells only 
expressed IgM protein ${ }^{62}$. Our data thus add to the current knowledge by showing that such transcript profile is found in a specific cell fraction and support a role for antigen involvement in MCL, as previous suggested ${ }^{59,61,63}$.

A subset of cells in patient 1, 3 and 7 was found to express the immature pro-B cell marker, SOX4, suggesting that not all MCL cells originate from mature, naive B cells and maybe some patients carry a reservoir of more immature malignant cells, which would support the hypothesis of multiple cellular origins of $\mathrm{MCL}^{61}$. Additionally, it suggests a potential clinical role for SOX4 to supplement one of the most important clinical MCL markers, and transcription factor homologue, SOX11. It is known that SOX4 is required for the development and differentiation of early B cells ${ }^{31}$. We observed that in MCL BM, the non-malignant pro-/pre-B cells were characterized by SOX4 expression, whereas the clinically defined blastoid MCL case (patient 1) was marked by a subset of cells expressing both SOX11 and SOX4 together with FAT1. Although further studies are required to establish its role in blastoid MCL, the latter was found to be exclusively expressed in this particular patient (20\% of SOX $11^{+}$ cells). FAT1 has been described as having both tumor suppressive ${ }^{64-68}$ and oncogenic ${ }^{69-72}$ roles, depending on the context. In the context of MCL, somatic mutations in the FAT1 gene have been reported in a few patients ${ }^{41}$, but its role in MCL has, to our knowledge, not yet been described. Interestingly, evidence points to FAT1 being a specific marker in acute lymphoblastic leukemia $(\mathrm{ALL})^{70,73}$. Additionally, the blastoid case presented here, also expressed the pre-B-ALL marker CD10 in a subset of SOX $11^{+}$cells. It is known that SOX4 plays a central role in the survival of malignant lymphoblasts ${ }^{34,35,74}$ and possibly predicts clinical outcome ${ }^{34}$. In cervical squamous cell carcinoma, FAT1 positively correlated with SOX4, and upregulated it to promote migration and invasion of cancer cells ${ }^{72}$. High FAT1 levels also predicted poor survival ${ }^{72}$. Thus, these markers, posed for further investigation, may help to establish the differentiation state and possibly prognosis of MCL. This raises the question of a possible prognostic value for the fraction of non-malignant SOX $4^{+}$or immature SOX $11^{+}$cells.

The clinical marker KI67, which is often employed for the prognostication of MCL, was restricted to a compartment of SOX $4^{+}$pro-/pre-B cells. Since CD19+ BM cells were sorted as singlets, cell doublets, probably including proliferating cells, were excluded therefore supporting the few number of KI67 positive MCL cells in the single cell data. Only in the blastoid case, a very small number of KI67 expressing malignant cells was found, which could be due to a high KI-67 staining index observed by immunohistochemistry for this blastoid MCL patient.

The samples with the highest quality (patient 2, 4, and 6) reflected the highest degree of MCL infiltration in the bone marrow samples and the highest spatial resolution. The transcriptional profile at the single-cell level is known to be noisier than bulk analyses ${ }^{75}$. This may partially be attributed to technical dropout in reverse transcription, extensive amplification of the small amount of RNA or may be caused by biological mechanisms, such as cell cycle or transcriptional bursting ${ }^{49,76}$. For this reason, the reported findings are preliminary and hypothesis-generating only, and must be further explored and confirmed.

In conclusion, our study confirms the inter-patient heterogeneity of MCL and provides insight into molecular pathology markers analyzed in MCL diagnostics at the single-cell transcription level. Importantly, the coinciding FAT1 and SOX4 mRNA expression in the SOX $11^{+}$cluster of malignant cells was specific for the blastoid case and may directly hold evidence of cells with a more immature profile and not just reflect a distinct morphology. Thus, it may be an important functional gene expression signature in this morphological subtype of MCL. We showed that SOX11 expression positively correlated with the mRNA expression of molecular pathology markers frequently applied in MCL diagnostics. Importantly, we identified a fraction of MCL cells expressing transcripts associated with antigen-experienced B cells in addition to CD23 positive cells, otherwise differentially associated with CLL, and co-expression of $\kappa$ and $\lambda$ Ig light chain genes.

\section{Materials and methods}

Mononuclear cells (MNCs) from 8 patients (62-88 years, Table 1) diagnosed with MCL at Odense University Hospital (OUH), Denmark, from 2017 to 2020, were isolated by Ficoll (GE Health Care, Chicago IL, USA) gradient centrifugation from bone marrow (BM) at diagnosis and either stored in RPMI medium (Gibco, Thermo Fisher Scientific, Waltham, MA, USA) with 20\% FBS (Gibco, Invitrogen, Thermo Fisher Scientific, Waltham, MA, USA) and 10\% DMSO (Sigma-Aldrich, St. Louis, MI, USA) in liquid nitrogen for subsequent cell isolation, or in mRNA lysis buffer (Roche, Basel, Switzerland) and stored at $-80^{\circ} \mathrm{C}$. All patients had nodal involvement, BM involvement, and were positive for both cyclin D1 and SOX11, determined by immunohistochemistry with an otherwise heterogeneous clinical presentation.

Single cell sample preparation and cell sorting. $\quad 2.76-10$ million cells from cryopreserved MNCs were stained with conjugated antibodies for CD19 (clone HIB19, BD Bioscience, Franklin Lakes, NJ, USA) and CD3 (clone SK7, BD Bioscience, Franklin Lakes, NJ, USA) in Hank's Balanced Salt Solution (HBSS; Gibco, Invitrogen, Thermofisher Scientific, Waltham MA, USA) 2\% FBS (Gibco, Invitrogen, Thermofisher Scientific, Waltham MA, USA) after blocking with Fc Receptor Block (BD Bioscence, Franklin Lakes, NJ, USA). Subsequently, cells were stained with Annexin V (Biolegend, San Diego, CA, USA) and 7AAD (BD Pharmingen, BD Bioscience, Franklin Lakes, NJ, USA) in Annexin V binding buffer (Biolegend, San Diego, CA, USA). The 7AAD Annexin $\mathrm{V}^{-} \mathrm{CD} 3{ }^{-} \mathrm{CD} 19^{+} \mathrm{B}$ cells were sorted (Fig. S1) on a FACS ARIA III (BD) using a $100 \mu \mathrm{m}$ nozzle and attained a purity of 75.6-99.7\% from singlet gate and 12.3-62.8\% from total (Suppl. Table S1, Fig. S2), indicating a higher fraction of apoptotic cells and debris in some samples.

When sufficient number of cells were available (> 15,000 events, 4/8 samples), viability was assessed with trypan blue (Sigma-Aldrich, St. Louis, Mi, USA) staining showing that the median percentage of viable cells was $92.9 \%$ (range $91.3-100 \%$ ). The sorted $7 \mathrm{AAD}^{-}$Annexin $\mathrm{V}^{-} \mathrm{CD} 3{ }^{-} \mathrm{CD} 19^{+} \mathrm{B}$ cells were fixated according to $10 \times$ Genomics protocol (Suppl. methods) and stored at $-80^{\circ} \mathrm{C}$ prior to sequencing. Fixated cells were rehydrated prior to single cell RNA sequencing according to the protocol from 10X Genomics (Suppl. methods). RNA integrity number (RIN) was assessed using Bioanalyzer RNA 6000 Pico Kit (Agilent Technologies, CA, USA) 
on an Agilent Bioanalyzer 2100, reaching RIN of 8.3-9.1 for patient 2-4 and 6, while unavailable for patient 1, 5,7 and 8 (Table S2).

Single cell RNA library preparation and sequencing. Cellular suspensions were aimed at 10,000 cells per sample loaded onto a Chromium Next GEM Chip G together with Next GEM Single Cell 3' v3.1 Gel Beads $(10 \times$ Genomics, Pleasanton, CA, USA) and partitioning oil to generate single cell Gel Beads-in-Emulsion (GEMs), followed by reverse transcription at $53^{\circ} \mathrm{C}$. GEMs were broken using Recovery Agent $(10 \times$ Genomics), and the resulting cDNA was cleaned up with DynaBeads MyOne Silane Beads (Thermo Fisher Scientific, Waltham, MA, USA) and amplified by PCR using Single Cell 3' GEM Kit v3.1 with subsequent cDNA cleanup (SPRIselect Reagent Beads, Beckman Coulter, Brea, CA, USA). Concentrations were measured with Qubit dsDNA HS Assay Kit (Thermo Fisher Scientific, Waltham, MA, USA). Enzymatic fragmentation, end-repair, and A-tailing were performed in one step using Single Cell 3' Library Kit v3.1, and were followed by a double-sided size selection using SPRIselect Reagent Beads. After a final double-sided size selection, the fragment sizes and concentrations were measured using QIAxcel DNA High Resolution Kit (1200) (Qiagen, Hilden, Germany) and KAPA Library Quantification Kit (Roche, Basel, Switzerland), respectively. Finally, the single-cell RNA (scRNA) libraries were sequenced on a NovaSeq 6000 (S1 Reagent Kits, Illumina, San Diego, CA, USA) platform, aiming at 40,000-60,000 reads per cell. Sequencing output per flow cell $(2 \times 50 \mathrm{bp})$ were 259 (sample 1-4) and $224 \mathrm{~Gb}$ (sample $5-8$ ) with $>90 \%$ of the base calls reaching a quality score of 30 or more.

Processing and analysis of single cell RNA sequencing data. Sequencing raw data demultiplexing was performed with Cell Ranger $m k f a s t q$ (Cell Ranger v3.1.0, 10x), and subsequent alignment to reference genome GRCh38 (prebuilt, 10x, GENCODE v32/Ensembl 98) was performed with STAR ${ }^{77}$ through Cell Ranger count. Merging of data from all patients and cross-sample normalization, as well as intra-/inter-sample differential expression analyses were performed in R (R 3.6, Seurat 3.2 ${ }^{78}$ ). Doublets, low quality cells and empty droplets were removed based on feature counts, mitochondrial read fraction and expression of $\mathrm{B}$ markers. The thresholds for the filtering were defined by Tukey's fences $( \pm 1.5 \mathrm{IQR})$ and outliers were removed from further analysis. Cells were transcriptionally restricted to positive expression of at least one of the following B-cell markers: IgH genes, Ig light chain genes, CD20, CD19 or CD79A/B. We defined positive expression of a given gene as more than $0.01 \%$ percent of counts originating from the specific feature, using Seurat function PercentageFeatureSet with regex pattern " $\wedge$ feature $\$$ "78,79. Multiple regression of molecular pathology markers frequently analyzed in diagnosis of MCL was performed in R, using the linear model ( $\mathrm{lm})$.

We combined the single cell transcriptomes of $\mathrm{CD} 19^{+} \mathrm{B}$ cells from all eight patients and jointly visualized these using Uniform Manifold Approximation and Projection (UMAP, Fig. 1A) for dimensional reduction of gene expression profiles to low-dimension feature space. Clustering of cells was performed with SNN clustering using cluster resolution 1.5 for the merged analysis and ranging from cluster resolution $0.2-0.5$ for analysis of individual samples, selected according to overall quality. Clusters of MCL cells were distinguished from nonmalignant $\mathrm{B}$ cells based on gene expression profiling (GSEA), monoclonality (restricted light chain expression) and expression of SOX11.

A total of 30,565 cells were sequenced (1018-6040 per sample) with mean reads per cell above 82,511 for 6 out of 8 samples (range 82,511-254,028 reads), while being lower, 25,422 and 33,591 mean reads per cell, for two samples (sample 5 and 8). The median unique molecular identifier counts per cell were 434-2704, while the median genes per cell was 309-1151 (Table S3). For samples 3, 5, and 7, the median genes per cell was less than 500 genes (309-460).

Sequencing of clonal rearrangements. DNA from MNCs was extracted using the MagNA LC DNA isolation kit (Roche), and quantification of DNA performed using the Qubit 2.0 dsDNA HS assay kit and a Qubit 2.0 fluorometer (Thermo Fisher Scientific). A minimum of $50 \mathrm{ng}$ DNA (50-78 ng) was used for next generation sequencing (NGS) of the immunoglobulin heavy chain clonal rearrangement using the LymphoTrack Dx IGH FR1 assay (Invivoscribe, San Diego, CA, USA) and a Prime Ion Gene Studio S5 sequencer (Ion Torrent; Thermo Fisher Scientific) according to the provided instructions. Data were analysed using the LymphoTrack Dx Software S5 package (Invivoscribe, San Diego, CA, USA). Each merged clonal sequence was evaluated for evidence of somatic hypermutation (SHM), as described by the supplier (Invivoscribe).

Ethical considerations. Informed consent was obtained from all patients. The project was approved by the National Committee on Health Research Ethics, Denmark (Approval No. 1605184), and data were handled in accordance with the requirements of the Danish Data Protection Authority.

\section{Data availability}

Sequencing data $(10 \times$ Cell Ranger output $)$ is available at https://doi.org/10.6084/m9.figshare.14743233. Please cite paper accordingly.

Received: 7 June 2021; Accepted: 7 September 2021

Published online: 27 September 2021

\section{References}

1. Jain, P. \& Wang, M. Mantle cell lymphoma: 2019 update on the diagnosis, pathogenesis, prognostication, and management. Am. J. Hematol. 94(6), 710-725 (2019). 
2. Veloza, L., Ribera-Cortada, I. \& Campo, E. Mantle cell lymphoma pathology update in the 2016 WHO classification. Ann. Lymphoma 3, 1-17 (2019).

3. Tsujimoto, Y. et al. Clustering of breakpoints on chromosome 11 in human B-cell neoplasms with the $t(11 ; 14)$ chromosome translocation. Nature 315(6017), 340-343 (1985).

4. Jares, P., Colomer, D. \& Campo, E. Genetic and molecular pathogenesis of mantle cell lymphoma: Perspectives for new targeted therapeutics. Nat. Rev. Cancer 7(10), 750-762 (2007).

5. Salaverria, I. et al. CCND2 rearrangements are the most frequent genetic events in cyclin D1(-) mantle cell lymphoma. Blood 121(8), 1394-1402 (2013).

6. Royo, C. et al. The complex landscape of genetic alterations in mantle cell lymphoma. Semin. Cancer Biol. 21(5), 322-334 (2011).

7. Gao, J., Peterson, L., Nelson, B., Goolsby, C. \& Chen, Y. H. Immunophenotypic variations in mantle cell lymphoma. Am. J. Clin. Pathol. 132(5), 699-706 (2009).

8. Jung, D. \& Alt, F. W. Unraveling V(D)J recombination; insights into gene regulation. Cell 116(2), 299-311 (2004).

9. Kiyoi, H. \& Naoe, T. Immunoglobulin variable region structure and B-cell malignancies. Int. J. Hematol. 73(1), 47-53 (2001).

10. Swerdlow, S. H. et al. The 2016 revision of the World Health Organization classification of lymphoid neoplasms. Blood 127(20), 2375-2390 (2016).

11. Navarro, A. et al. Molecular subsets of mantle cell lymphoma defined by the IGHV mutational status and SOX11 expression have distinct biologic and clinical features. Can. Res. 72(20), 5307-5316 (2012).

12. Jares, P., Colomer, D. \& Campo, E. Molecular pathogenesis of mantle cell lymphoma. J. Clin. Investig. 122(10), 3416-3423 (2012).

13. Ek, S., Dictor, M., Jerkeman, M., Jirström, K. \& Borrebaeck, C. A. Nuclear expression of the non B-cell lineage Sox 11 transcription factor identifies mantle cell lymphoma. Blood $111(2), 800-805$ (2008).

14. Mozos, A. et al. SOX11 expression is highly specific for mantle cell lymphoma and identifies the cyclin D1-negative subtype. Haematologica 94(11), 1555-1562 (2009).

15. Fernàndez, V. et al. Genomic and gene expression profiling defines indolent forms of mantle cell lymphoma. Can. Res. 70(4), 1408-1418 (2010).

16. Royo, C. et al. Non-nodal type of mantle cell lymphoma is a specific biological and clinical subgroup of the disease. Leukemia 26(8), 1895-1898 (2012)

17. Hoster, E. et al. Prognostic value of Ki-67 index, cytology, and growth pattern in mantle-cell lymphoma: results from randomized trials of the european mantle cell lymphoma network. J. Clin. Oncol. Off. J. Am. Soc. Clin. Oncol. 34(12), 1386-1394 (2016).

18. Shrestha, R., Bhatt, V. R., Guru Murthy, G. S. \& Armitage, J. O. Clinicopathologic features and management of blastoid variant of mantle cell lymphoma. Leuk. Lymphoma 56(10), 2759-2767 (2015).

19. Dy, P. et al. The three SoxC proteins-Sox4, Sox11 and Sox12-exhibit overlapping expression patterns and molecular properties. Nucleic Acids Res. 36(9), 3101-3117 (2008).

20. Hoser, M. et al. Sox12 deletion in the mouse reveals nonreciprocal redundancy with the related Sox4 and Sox 11 transcription factors. Mol. Cell. Biol. 28(15), 4675-4687 (2008).

21. Jiang, Y. et al. Transcription factors SOX4 and SOX11 function redundantly to regulate the development of mouse retinal ganglion cells. J. Biol. Chem. 288(25), 18429-18438 (2013).

22. Penzo-Méndez, A. I. Critical roles for SoxC transcription factors in development and cancer. Int. J. Biochem. Cell Biol. 42(3), $425-428$ (2010).

23. Palomero, J. et al. SOX11 promotes tumor angiogenesis through transcriptional regulation of PDGFA in mantle cell lymphoma. Blood 124(14), 2235-2247 (2014).

24. Balsas, P. et al. SOX11 promotes tumor protective microenvironment interactions through CXCR4 and FAK regulation in mantle cell lymphoma. Blood 130(4), 501-513 (2017).

25. Kuo, P. Y. et al. SOX11 augments BCR signaling to drive MCL-like tumor development. Blood 131(20), 2247-2255 (2018).

26. Palomero, J. et al. SOX11 defines two different subtypes of mantle cell lymphoma through transcriptional regulation of BCL6. Leukemia 30(7), 1596-1599 (2016).

27. Vegliante, M. C. et al. SOX11 regulates PAX5 expression and blocks terminal B-cell differentiation in aggressive mantle cell lymphoma. Blood 121(12), 2175-2185 (2013).

28. Wegner, M. From head to toes: The multiple facets of Sox proteins. Nucleic Acids Res. 27(6), 1409-1420 (1999).

29. van de Wetering, M., Oosterwegel, M., van Norren, K. \& Clevers, H. Sox-4, an Sry-like HMG box protein, is a transcriptional activator in lymphocytes. EMBO J. 12(10), 3847-3854 (1993).

30. Smith, E. \& Sigvardsson, M. The roles of transcription factors in B lymphocyte commitment, development, and transformation. J. Leukoc. Biol. 75(6), 973-981 (2004).

31. Sun, B. et al. Sox4 is required for the survival of pro-B cells. J. Immunol. (Baltimore Md. 1950) 190(5), 2080-2089 (2013).

32. Lu, J. W. et al. Overexpression of SOX4 correlates with poor prognosis of acute myeloid leukemia and is leukemogenic in zebrafish. Blood Cancer J. 7(8), e593 (2017).

33. Omidvar, N. et al. PML-RARa co-operates with Sox4 in acute myeloid leukemia development in mice. Haematologica 98(3), 424-427 (2013).

34. Ramezani-Rad, P. et al. SOX4 enables oncogenic survival signals in acute lymphoblastic leukemia. Blood 121(1), 148-155 (2013).

35. Ma, H. et al. The Sox4/Tcf7l1 axis promotes progression of BCR-ABL-positive acute lymphoblastic leukemia. Haematologica 99(10), 1591-1598 (2014)

36. Vervoort, S. J., van Boxtel, R. \& Coffer, P. J. The role of SRY-related HMG box transcription factor 4 (SOX4) in tumorigenesis and metastasis: Friend or foe?. Oncogene 32(29), 3397-3409 (2013).

37. Shaffer, A. L., Rosenwald, A. \& Staudt, L. M. Lymphoid malignancies: The dark side of B-cell differentiation. Nat. Rev. Immunol. 2(12), 920-932 (2002).

38. Pérez-Galán, P., Dreyling, M. \& Wiestner, A. Mantle cell lymphoma: Biology, pathogenesis, and the molecular basis of treatment in the genomic era. Blood 117(1), 26-38 (2011).

39. Hansen, M. H. et al. Molecular characterization of sorted malignant B cells from patients clinically identified with mantle cell lymphoma. Exp. Hematol. 84, 7-18 (2020).

40. Bea, S. et al. Landscape of somatic mutations and clonal evolution in mantle cell lymphoma. Proc. Natl. Acad. Sci. U.S.A. 110(45), $18250-18255$ (2013).

41. Wu, C. et al. Genetic heterogeneity in primary and relapsed mantle cell lymphomas: Impact of recurrent CARD11 mutations. Oncotarget 7(25), 38180-38190 (2016).

42. Landau, D. A. et al. Evolution and impact of subclonal mutations in chronic lymphocytic leukemia. Cell 152(4), 714-726 (2013).

43. Liu, F. et al. Clonal heterogeneity of mantle cell lymphoma revealed by array comparative genomic hybridization. Eur. J. Haematol. 90(1), 51-58 (2013).

44. Becht, E. et al. Dimensionality reduction for visualizing single-cell data using UMAP. Nat. Biotechnol. 37(1), 38-44 (2018).

45. Hay, S. B., Ferchen, K., Chetal, K., Grimes, H. L. \& Salomonis, N. The Human Cell Atlas bone marrow single-cell interactive web portal. Exp. Hematol. 68, 51-61 (2018).

46. Haddad, R. et al. Molecular characterization of early human T/NK and B-lymphoid progenitor cells in umbilical cord blood. Blood 104(13), 3918-3926 (2004). 
47. Zhang, S. et al. Longitudinal single-cell profiling reveals molecular heterogeneity and tumor-immune evolution in refractory mantle cell lymphoma. Nat. Commun. 12(1), 2877 (2021).

48. Wang, L., Mo, S., Li, X., He, Y. \& Yang, J. Single-cell RNA-seq reveals the immune escape and drug resistance mechanisms of mantle cell lymphoma. Cancer Biol. Med. 17(3), 726-739 (2020).

49. Suter, D. M. et al. Mammalian genes are transcribed with widely different bursting kinetics. Science (New York, NY). 332(6028), 472-474 (2011).

50. Raj, A., Peskin, C. S., Tranchina, D., Vargas, D. Y. \& Tyagi, S. Stochastic mRNA synthesis in mammalian cells. PLoS Biol. 4(10), e309 (2006)

51. Larsson, A. J. M. et al. Genomic encoding of transcriptional burst kinetics. Nature 565(7738), 251-254 (2019).

52. Xu, D. Dual surface immunoglobulin light-chain expression in B-cell lymphoproliferative disorders. Arch. Pathol. Lab. Med. 130(6), 853-856 (2006).

53. Jiang, A. S. et al. Plasma cell myeloma with dual expression of kappa and lambda light chains. Int. J. Clin. Exp. Pathol. 11(9), $4718-4723(2018)$

54. Pauza, M. E., Rehmann, J. A. \& LeBien, T. W. Unusual patterns of immunoglobulin gene rearrangement and expression during human B cell ontogeny: Human B cells can simultaneously express cell surface kappa and lambda light chains. J. Exp. Med. 178(1), 139-149 (1993).

55. Giachino, C., Padovan, E. \& Lanzavecchia, A. kappa+lambda+ dual receptor B cells are present in the human peripheral repertoire. J. Exp. Med. 181(3), 1245-1250 (1995).

56. Schlette, E., Fu, K. \& Medeiros, L. J. CD23 expression in mantle cell lymphoma: Clinicopathologic features of 18 cases. Am. J. Clin. Pathol. 120(5), 760-766 (2003).

57. Saksena, A. et al. CD23 expression in mantle cell lymphoma is associated with CD200 expression, leukemic non-nodal form, and a better prognosis. Hum. Pathol. 89, 71-80 (2019).

58. Klapper, W. et al. Immunoglobulin class-switch recombination occurs in mantle cell lymphomas. J. Pathol. 209(2), 250-257 (2006).

59. Xochelli, A. et al. Molecular evidence for antigen drive in the natural history of mantle cell lymphoma. Am. J. Pathol. 185(6), $1740-1748$ (2015).

60. Babbage, G. et al. Mantle cell lymphoma with $\mathrm{t}(11 ; 14)$ and unmutated or mutated VH genes expresses AID and undergoes isotype switch events. Blood 103(7), 2795-2798 (2004).

61. Pouliou, E. et al. Numerous ontogenetic roads to mantle cell lymphoma: Immunogenetic and immunohistochemical evidence. Am. J. Pathol. 187(7), 1454-1458 (2017).

62. Malisan, F. et al. B-chronic lymphocytic leukemias can undergo isotype switching in vivo and can be induced to differentiate and switch in vitro. Blood 87(2), 717-724 (1996).

63. Gemenetzi, K. et al. B cell receptor immunogenetics in B Cell lymphomas: Immunoglobulin genes as key to ontogeny and clinical decision making. Front. Oncol. 10, 67 (2020).

64. Nakaya, K. et al. Identification of homozygous deletions of tumor suppressor gene FAT in oral cancer using CGH-array. Oncogene 26(36), 5300-5308 (2007).

65. Chen, M. et al. FAT1 inhibits the proliferation and metastasis of cervical cancer cells by binding $\beta$-catenin. Int. J. Clin. Exp. Pathol. 12(10), 3807-3818 (2019).

66. Chosdol, K. et al. Frequent loss of heterozygosity and altered expression of the candidate tumor suppressor gene "FAT" in human astrocytic tumors. BMC Cancer 9, 5 (2009).

67. Martin, D. et al. Assembly and activation of the Hippo signalome by FAT1 tumor suppressor. Nat. Commun. 9(1), 2372 (2018).

68. Morris, L. G. et al. Recurrent somatic mutation of FAT1 in multiple human cancers leads to aberrant Wnt activation. Nat. Genet. 45(3), 253-261 (2013).

69. Sadeqzadeh, E. et al. Dual processing of FAT1 cadherin protein by human melanoma cells generates distinct protein products. J. Biol. Chem. 286(32), 28181-28191 (2011).

70. de Bock, C. E. et al. The Fat 1 cadherin is overexpressed and an independent prognostic factor for survival in paired diagnosisrelapse samples of precursor B-cell acute lymphoblastic leukemia. Leukemia 26(5), 918-926 (2012).

71. Kwaepila, N., Burns, G. \& Leong, A. S. Immunohistological localisation of human FAT1 (hFAT) protein in 326 breast cancers. Does this adhesion molecule have a role in pathogenesis?. Pathology 38(2), 125-131 (2006).

72. Zhang, Y. \& Xu, H. LncRNA FAL1 upregulates SOX4 by downregulating miR-449a to promote the migration and invasion of cervical squamous cell carcinoma (CSCC) cells. Reprod. Sci. (Thousand Oaks, Calif) 27(3), 935-939 (2020).

73. de Bock, C. E. et al. T-cell acute lymphoblastic leukemias express a unique truncated FAT1 isoform that cooperates with NOTCH1 in leukemia development. Haematologica 104(5), e204-e207 (2019).

74. Neumann, M. et al. FAT1 expression and mutations in adult acute lymphoblastic leukemia. Blood Cancer J. 4, e224 (2014).

75. Marinov, G. K. et al. From single-cell to cell-pool transcriptomes: Stochasticity in gene expression and RNA splicing. Genome Res. 24(3), 496-510 (2014).

76. Kharchenko, P. V., Silberstein, L. \& Scadden, D. T. Bayesian approach to single-cell differential expression analysis. Nat. Methods 11(7), 740-742 (2014).

77. Dobin, A. \& Gingeras, T. R. Mapping RNA-seq reads with STAR. Curr. Protoc. Bioinform. 51, 11-14 (2015).

78. Butler, A., Hoffman, P., Smibert, P., Papalexi, E. \& Satija, R. Integrating single-cell transcriptomic data across different conditions, technologies, and species. Nat. Biotechnol. 36(5), 411-420 (2018).

79. Stuart, T. et al. Comprehensive integration of single-cell data. Cell 177(7), 1888-902.e21 (2019).

\section{Acknowledgements}

We thank Amplexa Genetics (Odense, Denmark) for performing single cell RNA sequencing of the samples. We are grateful to Hasselbalch \& Lykkegaard Andersens Forskningsfond, Harboefonden, Tømrermester Jørgen Holm og Hustru Elisa F Hansens Mindelegat, Dagmar Marshalls Fond, Else og Mogens Wedell-Wedelsborgs Legat, Dansk Lymfom gruppes legat, Fonden til Lægevidenskabens Fremme af A. P. Møller, Fabrikant Einar Willumsens Mindelegat and Fhv. Dir. Leo Nielsen og Hustru Karen Margrethe Nielsens legat for Lægevidenskabelig Grundforskning for having supported this project. Special thanks to Vickie Svane Kristensen for proof-reading and to Nina Friis Jensen and to Elisabeth Luna Højlund for technical assistance prior to sequencing of clonal IGH rearrangements.

\section{Author contributions}

C.G.N., S.V.H., M.H.H. and O.C. designed the study. S.V.H. and O.C. performed cell sorting and sequencing of clonal I.G.H. rearrangements, and S.V.H. prepared samples for single cell RNA sequencing. Data analysis was performed by M.H.H. and S.V.H., who also wrote the first draft of the manuscript. J.H. and M.B.M. was involved in clinical characterization of patient samples. J.H., M.B.M. and N.A. assisted the interpretation of clinical data. All authors reviewed, edited and approved the manuscript. 


\section{Competing interests}

The authors declare no competing interests.

\section{Additional information}

Supplementary Information The online version contains supplementary material available at https://doi.org/ 10.1038/s41598-021-98560-1.

Correspondence and requests for materials should be addressed to S.V.H.

Reprints and permissions information is available at www.nature.com/reprints.

Publisher's note Springer Nature remains neutral with regard to jurisdictional claims in published maps and institutional affiliations.

(c) (1) Open Access This article is licensed under a Creative Commons Attribution 4.0 International License, which permits use, sharing, adaptation, distribution and reproduction in any medium or format, as long as you give appropriate credit to the original author(s) and the source, provide a link to the Creative Commons licence, and indicate if changes were made. The images or other third party material in this article are included in the article's Creative Commons licence, unless indicated otherwise in a credit line to the material. If material is not included in the article's Creative Commons licence and your intended use is not permitted by statutory regulation or exceeds the permitted use, you will need to obtain permission directly from the copyright holder. To view a copy of this licence, visit http://creativecommons.org/licenses/by/4.0/.

(C) The Author(s) 2021 